\title{
Reduction in erosive tooth wear using stannous fluoride-containing dentifrices: a meta-analysis
}

\section{Giorgio Aldigueri TRENTIN(a) Laura Teixeira MENDES(a) \\ Bruna Soares da SILVA(a) \\ Luciano CASAGRANDE(a) \\ Fernando Borba de ARAUJO(a) \\ Tathiane Larissa LENZ|(a)}

(a) Universidade Federal do Rio Grande do Sul - UFRGS, School of Dentistry, PostGraduate Program in Pediatric Dentistry, Porto Alegre, RS, Brazil.

Declaration of Interests: The authors certify that they have no commercial or associative interest that represents a conflict of interest in connection with the manuscript.

\section{Corresponding Author:}

Tathiane Larissa Lenzi

E-mail: tathilenzi@hotmail.com

Submitted: June 23, 2020

Accepted for publication: March 15, 2021

Last revision: April 25, 2021

\begin{abstract}
Dentifrices containing different active agents may be helpful to allow rehardening and to increase the resistance of the eroded surface to further acids or mechanical impacts. This study aimed to compare the effects of conventional (sodium fluoride $[\mathrm{NaF}]$ ) and stannous fluoride $\left(\mathrm{SnF}_{2}\right)$-containing dentifrices on reducing erosive tooth wear (ETW). The PubMed/MEDLINE, Scopus, LILACS, BBO, EMBASE, TRIP electronic databases, and grey literature were searched until January 2021 to retrieve relevant in vitro and in situ studies related to research question. There were no restrictions on publication year or language. Two authors independently selected the studies, extracted the data, and assessed the risk of bias. ETW data were pooled to calculate and compare both dentifrices (overall analysis) and in vitro and in situ studies separately (subgroup analysis). Statistical analyses were performed using RevMan5.3 with a random effects model. Of 820 potentially eligible studies, 101 were selected for full-text analysis, and 8 were included in the systematic review and meta-analysis. There was a significant difference between $\mathrm{SnF}_{2}$-containing dentifrices and $\mathrm{NaF}$ dentifrices only for in vitro studies $(\mathrm{p}=0.04)$, showing a higher effect of the $\mathrm{SnF}_{2}$-containing dentifrices against the erosion/abrasion (effect size: -6.80 95\%CI: -13.42; -0.19). Most in vitro and in situ studies had high and low risk of bias, respectively. In vitro literature suggests that the ETW reduction is greater when using $\mathrm{SnF}_{2}$-containing dentifrices instead $\mathrm{NaF}$-containing dentifrices. However, the evidence level is insufficient for definitive conclusions. Clinical trials are necessary for a better understanding of the effect of these compounds on ETW.
\end{abstract}

Keywords: Tooth Erosion; Tooth Abrasion; Tin Fluorides; Fluoride Dentifrices; Systematic Review.

\section{Introduction}

Dental erosion is the chemical loss of mineralized tooth substance caused by the exposure to acid not derived from oral bacteria. ${ }^{1}$ Erosive demineralization is accompanied by softening of the tooth surfaces, resulting in increased susceptibility to abrasion, especially immediately after an erosive challenge. ${ }^{2,3}$ Dentifrices containing different active agents may be helpful in facilitating re-hardening or in increasing surface resistance to further acidic or mechanical insults. ${ }^{4}$ 
The main active ingredient found in dentifrices is fluoride. It is believed that the mechanism of action of fluoride against erosion occurs by inducing the formation of a layer on the eroded surface composed of $\mathrm{CaF}_{2}$ (in the case of conventional compounds, such as sodium fluoride - $\mathrm{NaF}$ or amine fluoride $\mathrm{AmF}$ ) or of metal-rich surface precipitates (in the case of stannous fluoride $-\mathrm{SnF}_{2}$ ). After application of $\mathrm{SnF}_{2}$, $\mathrm{Sn}$ is not only established onto but also incorporated into the enamel and dentin, which can lead to increased fluoride uptake and higher resistance to acids. ${ }^{5}$ Thus, these formulations are currently more promising compared to conventional fluorides. It is believed that the effect of $\mathrm{SnF}_{2}-$ containing formulations is more strongly associated with reduction in the enamel surface loss than with re-hardening of softened enamel. ${ }^{6}$

To the best of our knowledge, none clinical trial evaluated the protective effect of $\mathrm{SnF}_{2}$-containing dentifrices against erosion/abrasion. In this sense, clinicians are taken to rely on their clinical experience and on data from in vitro ${ }^{7-11}$ and in situ ${ }^{12-14}$ studies for decision-making. Most laboratory studies ${ }^{7-14}$ compared the helpful effects of use of $\mathrm{SnF}_{2}$-containing dentifrices formulated with and without $\mathrm{NaF}$ with $\mathrm{NaF}$-containing dentifrices on erosive wear. Pooled data of laboratory studies may determine the potential for clinical applicability of the fluoridated dentifrices against initial enamel erosion and abrasion.

Therefore, this study aimed systematically review the literature to investigate the effect of $\mathrm{SnF}_{2}$-containing dentifrices formulated with and without $\mathrm{NaF}$ in the reduction of erosive tooth wear compared with NaF-containing dentifrices.

\section{Methodology}

This study was reported according to the Preferred Reporting Items for Systematic Reviews and Meta-Analyses (PRISMA) statement. ${ }^{15}$

\section{Research Question}

The following research question was formulated to address the literature and outline the search strategy: "Are $\mathrm{SnF}_{2}$-containing dentifrices formulated with and without $\mathrm{NaF}$ more effective in reducing erosive tooth wear in primary or permanent dentition compared to NaF-containing dentifrices?"

Population: erosive tooth wear in the primary or permanent dentition

Intervention: $\mathrm{SnF}_{2}$-containing dentifrices formulated with and without $\mathrm{NaF}$

Comparison: NaF-containing dentifrices

Outcome: reduction of erosive tooth wear

Study design: laboratory studies (in vitro or in situ).

\section{Search strategy}

A comprehensive literature search of the PubMed/ MEDLINE, Scopus, LILACS, BBO and EMBASE databases was performed. Turning Research Into Practice (TRIP) database was used as an additional source of information. The grey literature was also explored using the database System for Information on Grey Literature in Europe (OpenGrey) and Google Scholar. The last search for potentially eligible studies was performed in January 2021. The subject search used a combination of controlled vocabulary and text words based on the search strategy for the PubMed/ MEDLINE database as follows:

)(()(()(()(()(()(()(()(()(()(()((Tooth, Deciduous[MeSH Terms]) OR tooth, deciduous) OR deciduous tooth) OR Dentition* , deciduous) OR deciduous Dentition*) OR Dentition*, primary) OR primary Dentition*) OR primary teeth) OR primary tooth) OR teeth, deciduous) OR deciduous teeth) OR teeth, primary) OR tooth, primary) OR primary tooth) OR Dentition, Permanent[MeSH Terms]) OR dentition, permanent) OR permanent dentition) OR dentition, secondary) OR secondary dentition) OR dentition, adult) OR adult dentition) AND Tooth Wear[MeSH Terms]) OR tooth Wear*) OR Wear* , tooth) OR dental Wear*) OR Wear* , dental) OR Tooth Erosion[MeSH Terms]) OR tooth Erosion*) OR Erosion*, tooth) OR dental Erosion*) OR erosive tooth wear)) AND Fluorides[MeSH Terms]) OR Fluoride* , Tin) OR Tin Fluoride*) OR Tin Difluoride*) OR Difluoride* , Tin) OR stannous Fluoride*) OR Fluoride*, stannous) OR cav-x) OR Floran*) OR Tin Tetrafluoride*) OR Tetrafluoride* , Tin) OR stannic Fluoride*) OR Fluoride* , stannic) OR gel-kam) OR gel-tin) OR omni i gel) OR omni med) OR Stanimax*) OR Fluoristan*) OR stannous fluoride) OR stannous)) AND $(((()((()(($ Sodium 
Fluoride[MeSH Terms]) OR sodium Fluoride*) OR Fluoride* , sodium) OR Zymafluor*) OR Fluoristat*) OR Ossin*) OR Calcium Fluoride[MeSH Terms]) OR calcium fluoride) OR fluoride, calcium) OR fluoride toothpaste) OR fluoride dentifrice)

A sensitive search strategy was adapted for the other databases. The results of searching the various sources were cross-checked to identify and eliminate duplicates. There were no restrictions on publication year or language.

\section{Selection and the inclusion and exclusion criteria}

The titles and abstracts of all retrieved studies were carefully assessed by two independent reviewers (B.S.S. and G.A.T.), who were previously trained and calibrated for study selection (Kappa $=0.95$ ). The studies were considered eligible if they were laboratory studies that evaluated the effect of $\mathrm{SnF}_{2}-$ containing dentifrices in the reduction of erosive tooth wear. The initial screening of titles and abstracts was performed using a standardized spreadsheet (Excel, Microsoft Corporation, Redmond, WA, USA). The references of all selected studies were manually searched for additional relevant studies that could fulfill the inclusion criteria.

The full texts of all studies that fulfilled the inclusion criteria for eligibility were then reviewed independently by the same reviewers. The exclusion criteria were: did not use a NaF-containing dentifrice as control; did not evaluate erosive tooth wear as outcome; did not use profilometry to measure surface loss; associated other erosion-preventive agent(s) to the use of dentifrice; did not use human or bovine teeth; and did not associate abrasion with erosion. Any disagreements regarding eligibility criteria were resolved by discussion with a third reviewer (L.T.M), and consensus was reached.

\section{Data extraction}

Data extraction was performed using a standardized spreadsheet (Excel, Microsoft Corporation, Redmond, WA, USA). For each study, the following data were systematically extracted: publication details (authors, country, and year); study methodology (study design, in vitro or in situ, sample size, erosion/abrasion cycles, composition, brand commercial and manufacturer of the dentifrices, and profilometry test); and outcome information (means and standard deviations of tissue loss values and percentage of surface loss).

\section{Risk of bias assessment}

Some authors independently evaluated the risk of bias based on and adapted from previous systematic reviews of in vitro ${ }^{16}$ and in situ ${ }^{17}$ studies. The following parameters were considered for in vitro studies: randomization of specimens; description of sample size calculation; and blinding of the operator of the testing machine and previous measurement of the enamel surface. For in situ studies, the parameters evaluated included: randomization of participants; description of sample size calculation; participant blinding; evaluator blinding; and previous measurements of the enamel surface. If the authors reported the parameter, the article received a "yes" for that specific parameter; if this information was not available or reported, the article received a "no." Articles that reported 1 or 2 items were classified as having a high risk of bias, 3 as medium risk, and 4 (in vitro studies) or 4-5 (in situ studies) as low risk. Disagreements between the reviewers regarding the classification of risk of bias were resolved by consensus.

\section{Data analyses}

For meta-analyses, pooled effect estimates were obtained by comparing means of tissue surface loss and respective standard deviations for $\mathrm{SnF}_{2}$-containing dentifrices, associated or not associated with $\mathrm{NaF}$, versus $\mathrm{NaF}$-containing dentifrices, irrespective of the study design, as well as considering subgroups (i.e., in vitro and in situ studies), separately. For studies that evaluated more than one dentifrice with the same concentration fluoride, the values were extracted and 1 mean was calculated using a formula according to the Cochrane Statistical Guidelines ${ }^{18}$ to obtain a single sample size and the mean and standard deviation values for both groups. In the selected studies, only data of interest were extracted and analyzed in the meta-analysis. Statistical differences between groups were calculated using RevMan version 5.3 (Review Manager, Cochrane Collaboration, Copenhagen, Denmark, 2014) using a random effect 
model. Differences with $p<0.05$ were considered to be statistically significant ( $Z$ test). Statistical heterogeneity among the studies was assessed using the Cochran's $Q$ test and inconsistency $\left(\mathrm{I}^{2}\right)$.

\section{Results}

\section{Search and selection}

Figure 1 depicts a flowchart summarizing the selection process for studies according to the PRISMA statement. ${ }^{15}$ The search strategy identified 820 potentially relevant records excluding duplicates. The first screening resulted in 101 studies remained for full-text reading. Finally, 8 papers were included in the systematic review and meta-analysis.

\section{Characteristics of the included studies}

A brief description of the data extracted from the included studies is described in Table 1. The articles were published between 2011 and 2019. Thirteen commercially available dentifrices were used, and two consisted of $\mathrm{SnF}_{2}$ and $\mathrm{NaF}$ in the composition, namely Pro-Expert Enamel protection $\left(\mathrm{SnF}_{2} 1100\right.$ parts per million [ppm] F + NaF 350 ppm F; Oral
B, Proctor \& Gamble; P\&G) and Pro-Health $\left(\mathrm{SnF}_{2}\right.$ 1100 ppm F + NaF 350 ppm F; Oral B). Considering the NaF-containing dentifrices, Crest ( $\mathrm{NaF} 1,500$ ppm F, P\&G) was the most tested. Crest ProHealth ${ }^{\mathrm{TM}}\left(\mathrm{SnF}_{2}\right.$ 1,100 ppm F; $P \& G$ ) was the $\mathrm{SnF}_{2}$-containing dentifrice more frequently evaluated in the included studies, and three studies, ${ }^{9,1011}$ used $\mathrm{SnF}_{2}$-containing gel (GelKam $\mathrm{SnF}_{2} 970$ ppm; Colgate Oral Pharmaceuticals). Included studies also evaluated dentifrices containing AmF/ $\mathrm{SnF}_{2,}{ }^{9,13} \mathrm{AmF} / \mathrm{NaF} / \mathrm{SnCl}_{2}$ (stannous chloride), ${ }^{7,9} \mathrm{NaF} /$ $\mathrm{SnCl}_{2}{ }^{9} \mathrm{TiF}_{4}$ (titanium fluoride) or $\mathrm{TiF}_{4} / \mathrm{NaF}{ }^{8}$

There was wide variability in the protocols used to simulate erosion/abrasion. The majority of studies $^{7,9-13}$ used citric acid solution; however, there was no consensus regarding acid concentrations, $\mathrm{pH}$ values, induction time, and mode of agitation of the solution. Moreover, the samples were brushed using an automated brushing machine in all in vitro ${ }^{7-11}$ studies. The brushing protocols varied considerately in the in situ studies. Two studies ${ }^{12,14}$ applied manual brushing of the lingual surface of the teeth for $30 \mathrm{~s}$, associated with mouthwash for $90 \mathrm{~s}$ twice per day, and one study ${ }^{13}$ used a slurry dentifrice immersion brushing protocol associated with a brushing machine.
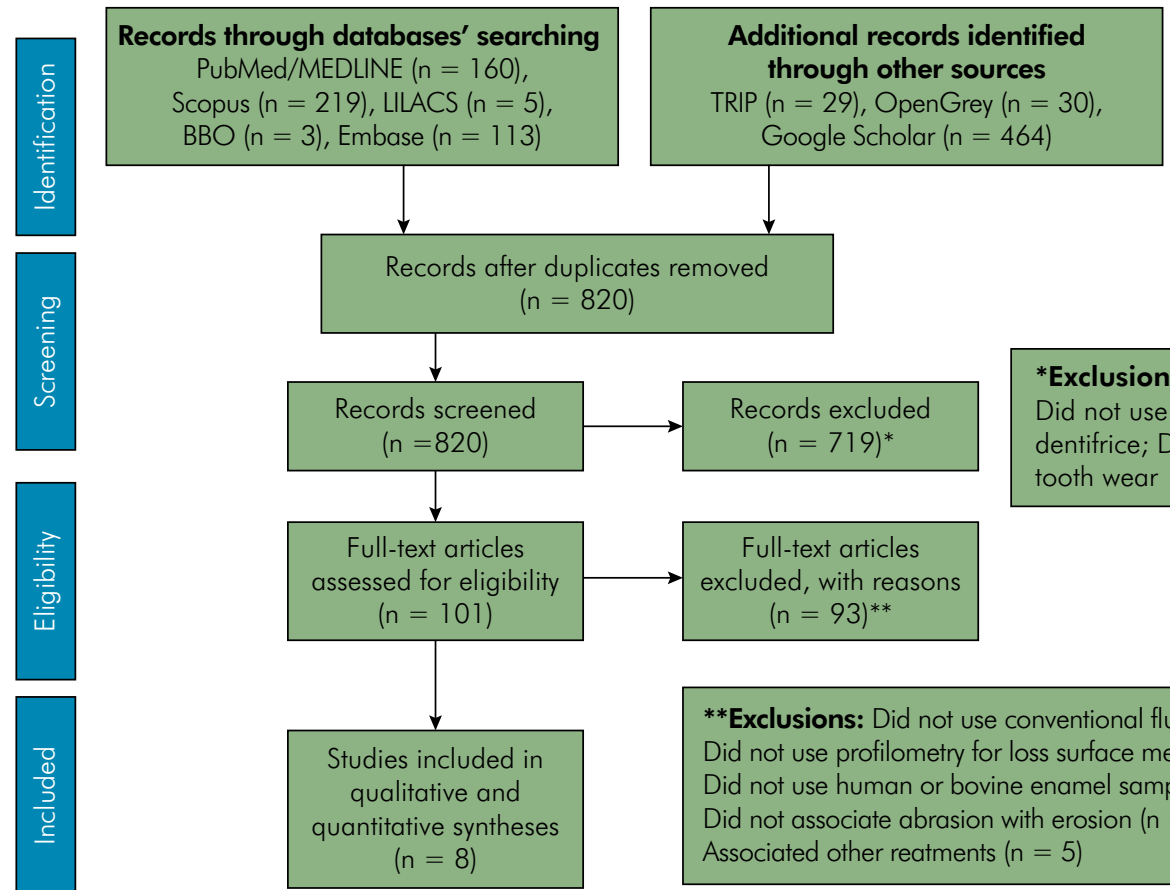

*Exclusions: Not in vitro or in situ study: Did not use stannous fluoride containing dentifrice; Did not evaluate erosive tooth wear

Records excluded $(n=719)^{*}$

Full-text articles excluded, with reasons $(n=93)^{* *}$

Figure 1. Flowchart diagram of study selection according to PRISMA statement. 


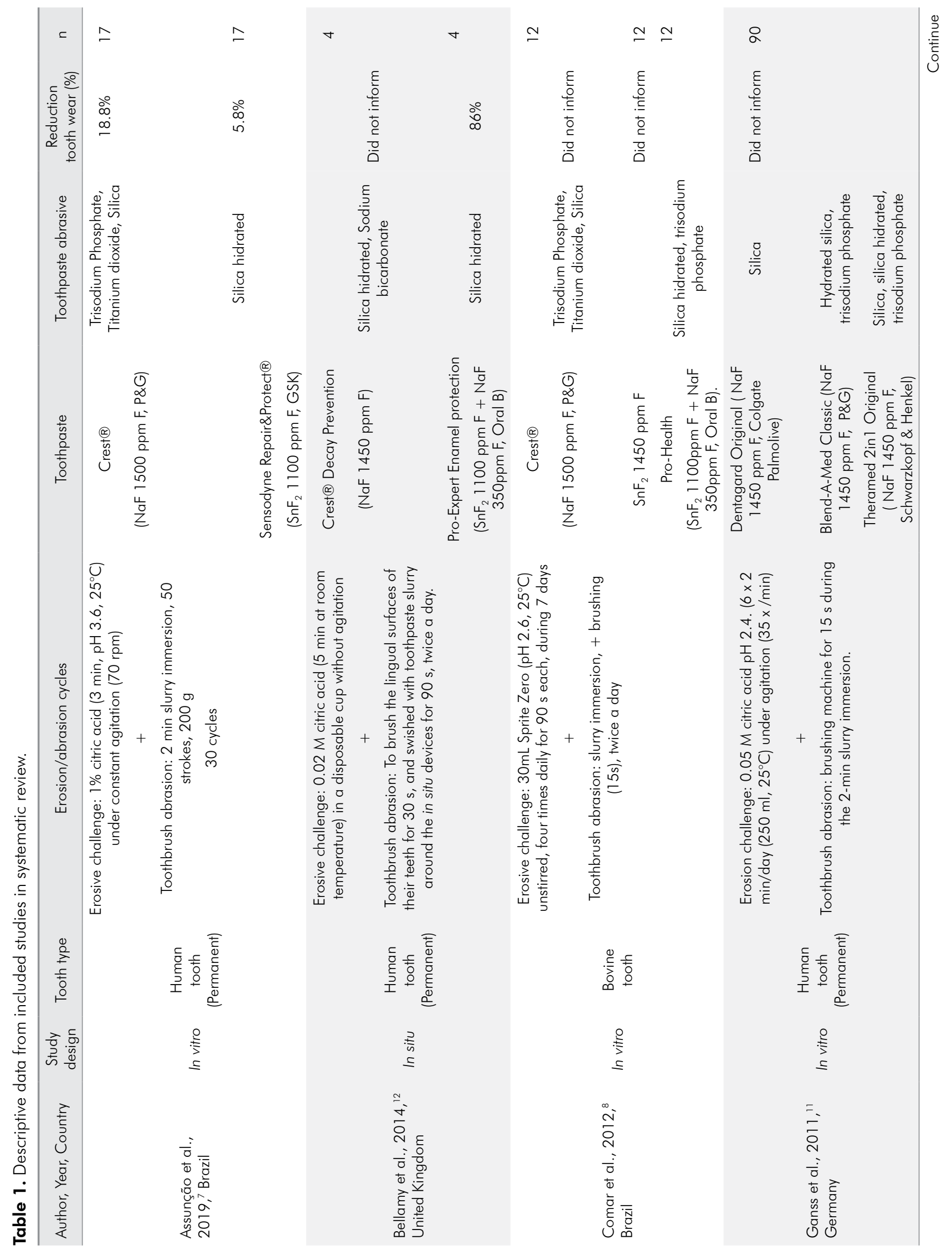


- Reduction in erosive tooth wear using stannous fluoride-containing dentifrices: a meta-analysis

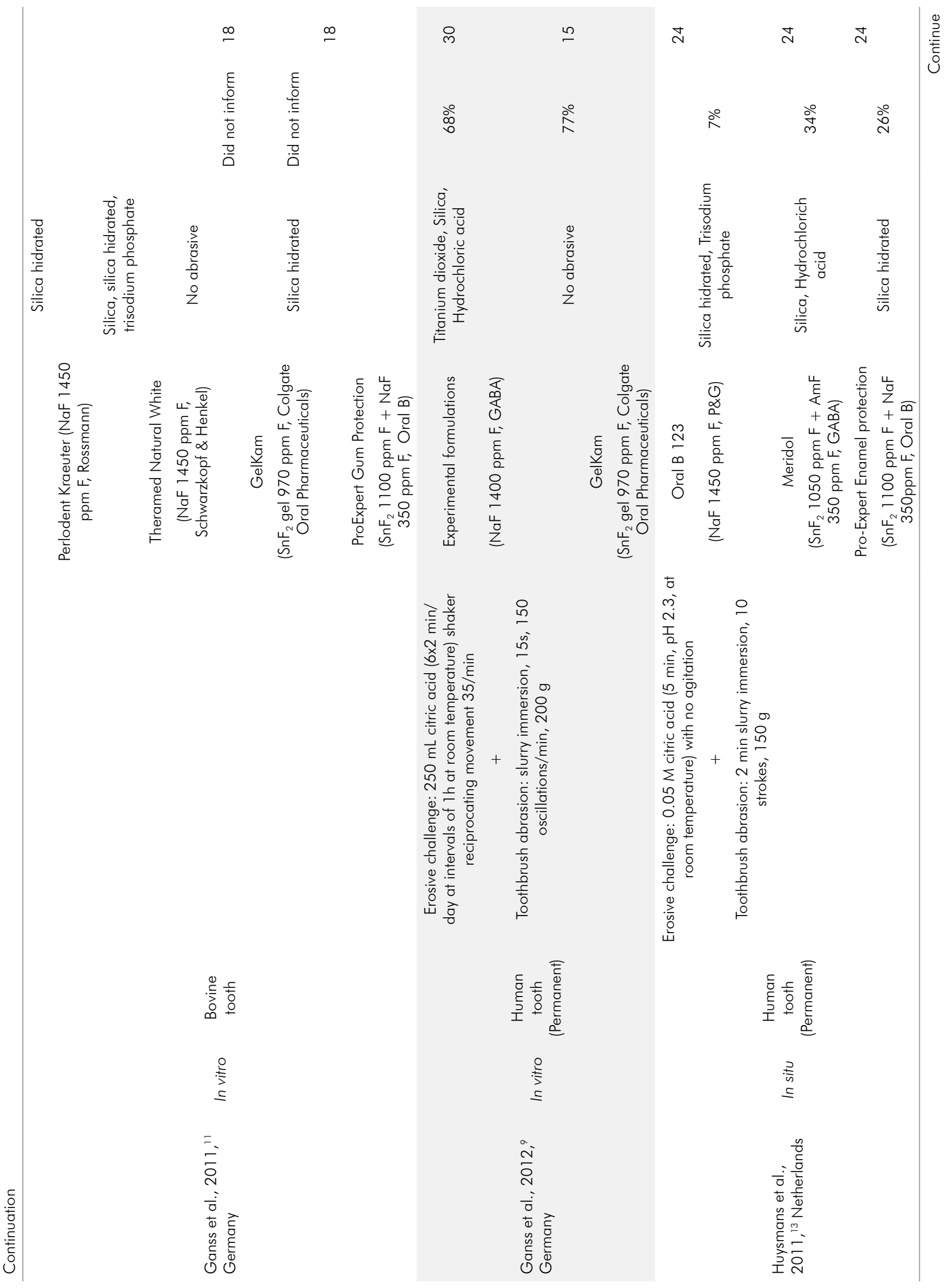




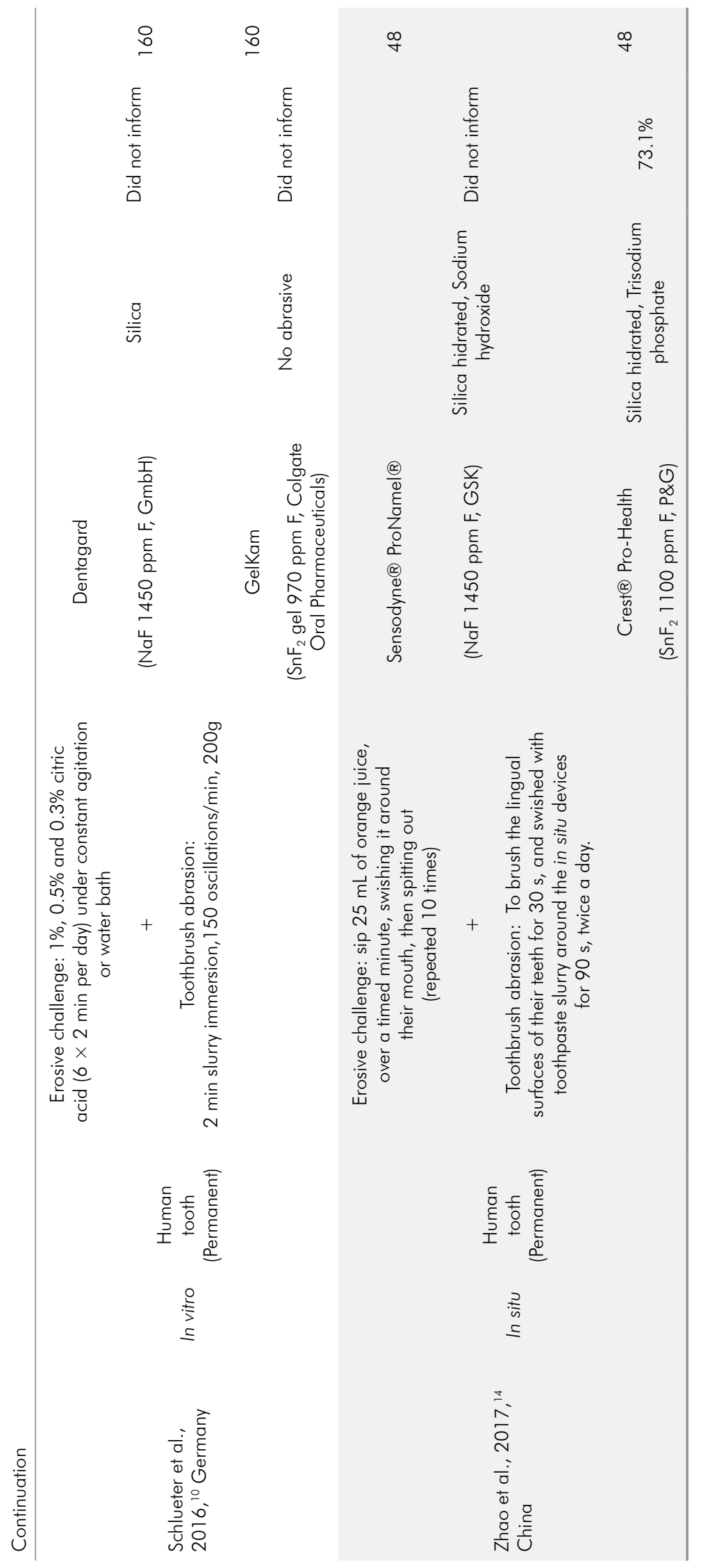




\section{Risk of bias}

Of the 5 in vitro studies included in the present review, 4 papers ${ }^{8,9,10,11}$ were scored as high risk of bias and one study ${ }^{7}$ as low risk of bias (Table 2). The item that received "no" most frequently was the blinding of the operator of the testing machine, and only 2 studies $^{7,10}$ reported the sample size calculation. Of the 3 in situ studies included, 2 papers ${ }^{13,14}$ were scored as low risk of bias and one study ${ }^{12}$ as medium (Table 3 ).
All studies reported the sample randomization, and only one study ${ }^{12}$ did not report enamel surface measurement before erosion induction.

\section{Data analyses}

The results of the meta-analysis comparing the reduction of erosive tooth wear by $\mathrm{SnF}_{2}$-containing dentifrices versus $\mathrm{NaF}$-containing dentifrices are shown in Figure 2. In the subgroup analysis, there

Table 2. Assessment of the risk of bias of included risk of bias studies in the systematic review.

\begin{tabular}{|c|c|c|c|c|c|}
\hline Study & Random & Sample size & Blinding & Measurement of enamel surface & Bias risk \\
\hline Assunção et al., $2019^{7}$ & Yes & Yes & Yes & Yes & Low \\
\hline Comar et al., $2012^{8}$ & Yes & No & No & Yes & High \\
\hline Ganss et al., $2011^{11}$ & No & No & No & No & High \\
\hline Ganss et al., $2012^{9}$ & No & No & No & No & High \\
\hline Schlveter et al., $2016^{10}$ & No & Yes & No & Yes & High \\
\hline
\end{tabular}

Random: randomization of specimens; sample size: description of sample size calculation; blinding: blinding of the operator of the testing machine; measurement of enamel surface: prior measurement of enamel surface.

Table 3. Assessment of the risk of bias of included studies in the systematic review.

\begin{tabular}{lcccccc}
\hline Study & Random & Sample size & $\begin{array}{c}\text { Blinding } \\
\text { participants }\end{array}$ & $\begin{array}{c}\text { Blinding } \\
\text { evaluator }\end{array}$ & $\begin{array}{c}\text { Prior measurement } \\
\text { of enamel surface }\end{array}$ & Bias risk \\
\hline Bellamy et al., 2014 2012 & Yes & Yes & No & Yes & No & Medium \\
Huysmans et al., $2011^{13}$ & Yes & No & Yes & Yes & Yes & Low \\
Zhao et al., 201714 & Yes & No & Yes & Yes & Yes & Low \\
\hline
\end{tabular}

Random: randomization of participants; sample size: description of sample size calculation; blinding: blinding of the participants and evaluator; measurement of enamel surface: prior measurement of enamel surface.

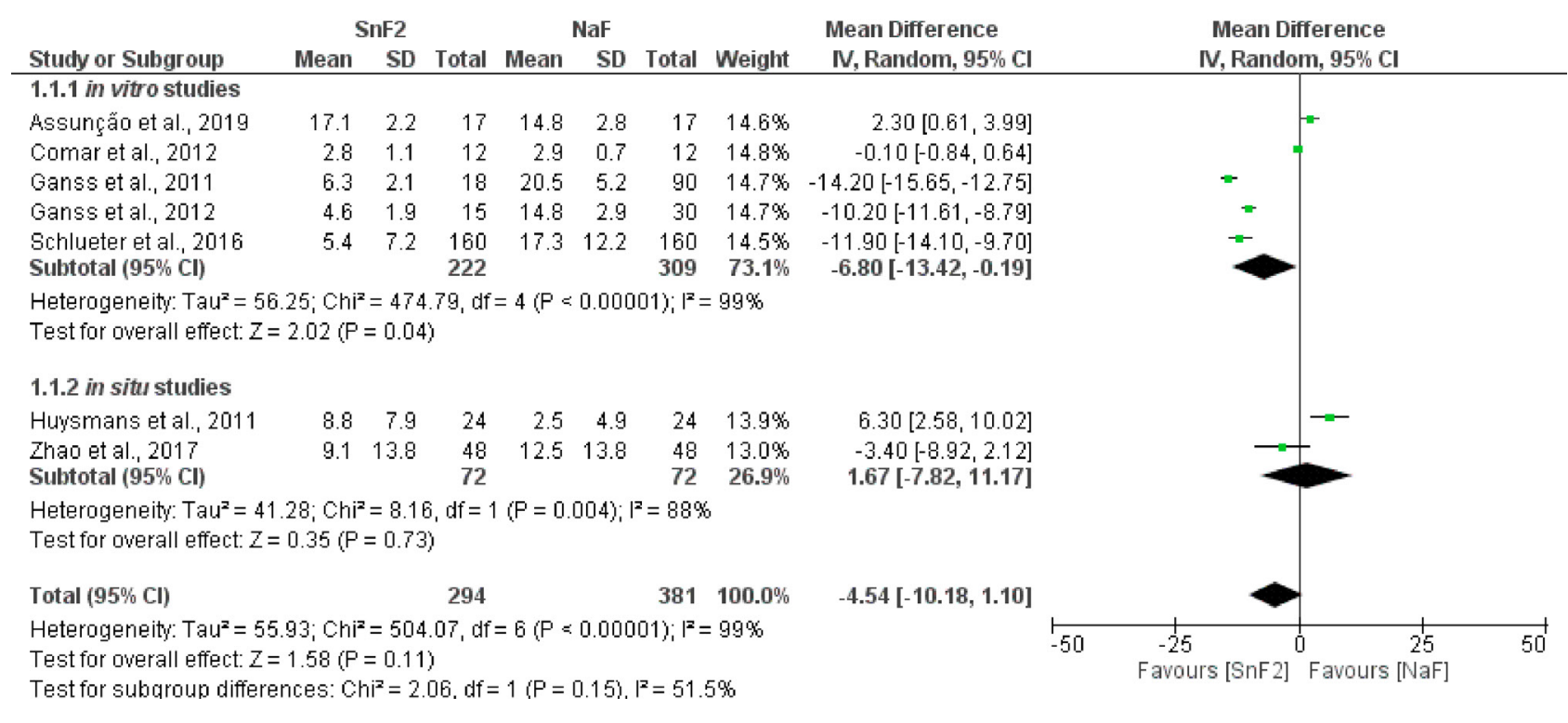

Figure 2. Summary findings of the meta-analyses comparing the use of $\mathrm{SnF}_{2}$-containing dentifricesversus NaF-containing dentifrices. 
was a significant difference between dentifrices for in vitro studies $(\mathrm{p}=0.04)$, showing evidence of a higher reduction of erosive tooth wear when using $\mathrm{SnF}_{2}$-containing dentifrices (effect size: -6.80 95\%CI: -13.42; -0.19). However, global analysis revealed that there was no significant difference between dentifrices $(p=0.11)$. The heterogeneity found was high $\left(I^{2}=99 \%\right)$.

Figure 3 summarizes the results of the metaanalysis comparing reduction of erosive tooth wear by $\mathrm{SnF}_{2} / \mathrm{NaF}$-containing dentifrices versus NaF-containing dentifrices. No difference was found between the groups ( $p=0.12)$, irrespective of the study design (in vitro studies, $\mathrm{p}=0.10$; in situ studies: $p=0.59$ ). The heterogeneity found was also high $\left(I^{2}=88 \%\right)$.

\section{Discussion}

There are several products available in the market that claim better erosive tooth wear protection. This is the first systematic review designed to determine whether $\mathrm{SnF}_{2}$-containing dentifrices are more effective in the reduction of erosive tooth wear compared with NaF-containing dentifrices. Profilometry is the method most used for surface loss assessment in laboratory studies. This method quantifies the loss of dental tissue in relation to a non-treated reference area in teeth subjected to erosive wear ${ }^{19}$. It is important to highlight that none dentifrice was capable of inhibiting erosive tooth wear. Nevertheless, meta-analyses results showed that there was a significant difference between $\mathrm{SnF}_{2}-$ containing dentifrices and $\mathrm{NaF}$-containing dentifrices only for in vitro studies ( $p=0.04)$, showing a higher effect of the $\mathrm{SnF}_{2}$-containing dentifrices against the erosion/abrasion (effect size: -6.80 95\%CI: -13.42; -0.19). This effect was not observed in situ studies, and it may be associated to smallest number of selected studies in relation to in vitro studies. The Sn-containing salts form a more resistant layer on the enamel surface, ${ }^{10}$ and it can interact with the salivary pellicle ${ }^{20}$ or be incorporated into the demineralized enamel surface, ${ }^{5}$ reducing the tooth wear provoked by the synergic effect of erosion and abrasion.

It has been suggested that surface precipitation of $\mathrm{CaF}_{2}$ plus the metallic layers make it more resistant not only to abrasion but also to further erosive insults ${ }^{7}$. Despite the absence of statistical differences, a higher mean difference (effect size, $-2.8495 \% \mathrm{CI}$ : $-6.45 ; 0.76]$ ) in the surface loss was found when NaF-containing dentifrices and $\mathrm{SnF}_{2} /$ NaF-containing dentifrices compared, favoring the latter. The effect of the underlying quality of evidence of the findings must be emphasized. Only a small number of studies were included,

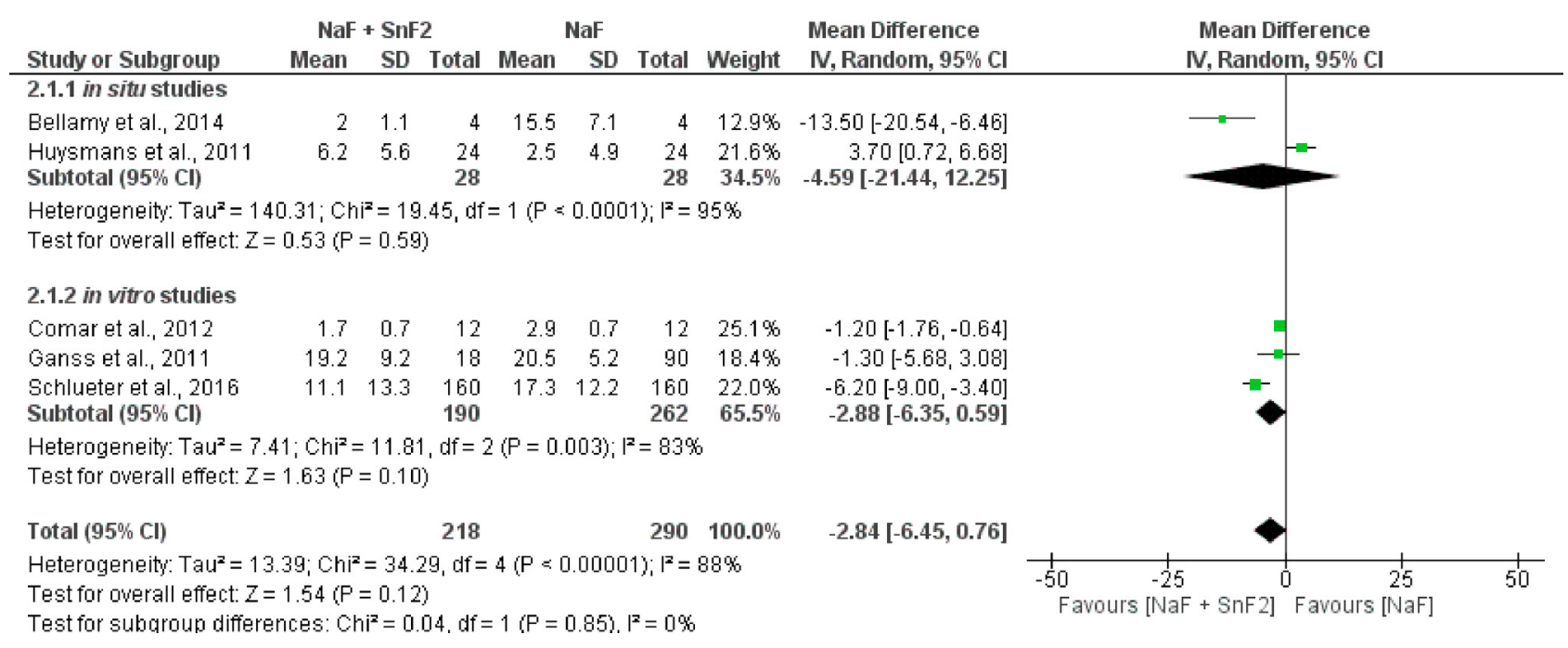

Figure 3. Summary findings of the meta-analyses comparing the use of $\mathrm{SnF}_{2} / \mathrm{NaF}$-containing dentifrices versus $\mathrm{NaF}$-containing dentifrices. 
and there was no consensus regarding with the erosion/abrasion protocol and dentifrices tested.

Although citric acid was used in most studies, there was wide variability regarding acid concentrations, $\mathrm{pH}$ values, induction time, and mode of agitation of the solution. It is known that changing the acid immersion movement from a water bath (smooth movement of the acid) to a shaker plate (jerky movement) increases enamel surface loss. ${ }^{10}$ It could be expected that toothbrushing would produce greater tissue loss in studies that used more concentrated citric acid and performed more dynamic movement conditions. ${ }^{10}$

It is important to bear in mind that other factors such as $\mathrm{pH}$, consistency and abrasivity ${ }^{21}$ may modulate the effect of fluoride dentifrices on dental erosion and abrasion. Due to acid contact, the tooth surface softens and becomes more prone to abrasion from toothbrushing ${ }^{3}$, especially immediately after an erosive challenge. In all selected studies, irrespective of the study design, acid exposure was immediately followed by an abrasion protocol. In attempts to optimize the preventive effect of the dentifrices, clinically, patients could increase waiting periods before brushing ${ }^{3}$ or could brush their teeth before rather than after an erosive attack. ${ }^{22}$ In addition, three studies ${ }^{9,10,11}$ included in this review used $\mathrm{SnF}_{2}$ gel, which did not contain abrasives in the formulation. A lower tooth surface loss was observed in these studies. Thus, evidence available so far suggests that $\mathrm{SnF}_{2}$-containing dentifrice gel seems be to the best option for reducing the harmful effects of the erosion and abrasion.

High heterogeneity seems to be almost unavoidable in laboratory studies, considering the methodological variability among them. The heterogeneity could have also occurred because small number of studies found. Moreover, they presented, in their majority, a small number of samples, and consequently high standard deviations, and a high number of covariables, favoring the heterogeneity. For statistical purposes, the fixed-and random-effect models were performed. Despite the small number of the included studies, ${ }^{23}$ the random effect was chosen based on the generalization inference, the intrinsic heterogeneity among studies, and assuming that each study estimates a different underlying true effect. ${ }^{24}$
To date, there is no standard and validated tool to assess the methodological quality of the in vitro and in situ studies. Thus, the risk of bias evaluation was based on and adapted from previous studies. ${ }^{16,17}$ Furthermore, inclusion and exclusion criteria were strictly defined as a way to avoid bias. Lack of clear information regarding sample size calculation, randomization, and blinding of the operator of the test machine was found in vitro studies. By contrast, randomization of participants and blinding of the participants and the evaluator(s) were aspects reported by all in situ studies included. Further studies should improve the conducting and reporting of laboratory testing using research reporting guidelines checklist in order to promote quality and transparency of evidence. It is likely that the results may have been influenced by publication bias, once negative results were probably not published or published in low-impact factor journals. Nonetheless, this aspect is present in all studies, not only laboratory studies. A broad search was used to try to overcome this problem, including grey literature. ${ }^{25}$ All included studies were performed in permanent teeth. One study ${ }^{7}$ evaluated the effect of dentifrices on surface loss permanent and primary teeth, but only data obtained in permanent teeth were considered. Since primary and permanent teeth present differences in enamel microstructure and composition, ${ }^{26}$ the results cannot be extrapolated to primary dentition. Moreover, only one study ${ }^{8}$ used bovine teeth. A sensitivity analysis was performed, and the removal of this study from the meta-analysis did not reduce the heterogeneity. Due to limited number of studies, this paper was retained in the analyses. The use of bovine teeth has been suggested as substitutes of human teeth in laboratory studies because the similarity on chemical composition, almost calcium and phosphorus content, ${ }^{27}$ enamel thickness ${ }^{28}$ and comparable acid resistance ${ }^{29}$ between bovine and human teeth.

The evaluation of products designed to treat clinically important oral care problems, such as dental erosion, is usually carried out using studies that come as closely as possible to the condition of interest. In vitro studies are the initial step in the evaluating products designed to help prevent or even 
reverse this condition. However, the findings cannot be extrapolated directly to the clinical conditions. Once demonstrated effective, the products can progress to more realistic modeling, such as in situ studies which models are used with minimal controlled exposure of teeth to common dietary acids to predict the potential efficacy of oral care products against erosive tooth wear. Clinical trials are necessary for a better understanding of the complex interaction of active ingredients and abrasives and their effects on erosive tooth wear, improving the evidence weak for clinical decision-making.

\section{Conclusion}

Despite a higher positive in vitro effect against the erosion/abrasion compared to NaF-containing dentifrices, the high heterogeneity and risk of bias in the included studies do not allow any definitive conclusion to be drawn.

\section{References}

1. Schlveter N, Amaechi BT, Bartlett D, Buzalaf MA, Carvalho TS, Ganss C, et al. Terminology of Erosive Tooth Wear: consensus report of a workshop organized by the ORCA and the Cariology Research Group of the IADR. Caries Res. 2020;54(1):2-6. https://doi.org/10.1159/000503308

2. Voronets J, Lussi A. Thickness of softened human enamel removed by toothbrush abrasion: an in vitro study. Clin Oral Investig. 2010 Jun;14(3):251-6. https://doi.org/10.1007/s00784-009-0288-y

3. Attin T, Siegel S, Buchalla W, Lennon ÁM, Hannig C, Becker K. Brushing abrasion of softened and remineralised dentin: an in situ study. Caries Res. 2004 Jan-Feb;38(1):62-6. https://doi.org/10.1159/000073922

4. Magalhães AC, Wiegand A, Buzalaf MA. Use of dentifrices to prevent erosive tooth wear: Harmful or helpful? Braz Oral Res. 2014;28(spe 1):1-6. https://doi.org/10.1590/S1806-83242013005000035

5. Comar LP, Souza BM, Al-Ahi LP, Martins J, Grizzo LT, Piasentim IS, et al. Mechanism of action of TiF4 on dental enamel surface: SEM/EDX, KOH-Soluble F, and X-Ray diffraction analysis. Caries Res. 2018 Jan;51(6):554-67. https://doi.org/10.1159/000479038

6. Lussi A, Carvalho TS. The future of fluorides and other protective agents in erosion prevention. Caries Res. 2015;49(1 Suppl 1):18-29. https://doi.org/10.1159/000380886

7. Assunção CM, Schlueter N, Rodrigues JA, Carvalho TS, Lussi A. Do fluoride toothpastes have similar preventive effect in permanent and primary teeth against erosive tooth wear? Int J Paediatr Dent. 2018 Nov;29:228-36. https://doi.org/10.1111/ipd.12449

8. Comar LP, Gomes MF, Ito N, Salomão PA, Grizzo LT, Magalhães AC. Effect of NaF, SnF2, and TiF4 toothpastes on bovine enamel and dentin erosion-abrasion in vitro. Int J Dent. 2012;2012:134350. https://doi.org/10.1155/2012/134350

9. Ganss C, von Hinckeldey J, Tolle A, Schulze K, Klimek J, Schlueter N. Efficacy of the stannous ion and a biopolymer in toothpastes on enamel erosion/abrasion. J Dent. 2012 Dec;40(12):1036-43. https://doi.org/10.1016/i.jdent.2012.08.005

10. Schlueter N, Lussi A, Tolle A, Ganss C. Effects of erosion protocol design on erosion/abrasion study outcome and on active Agent (NaF and SnF 2) efficacy. Caries Res. 2016;50(2):170-9. https://doi.org/10.1159/000445169

11. Ganss C, Lussi A, Grunau O, Klimek J, Schlueter N. Conventional and anti-erosion fluoride toothpastes: effect on enamel erosion and erosion-abrasion. Caries Res. 2011;45(6):581-9. https://doi.org/10.1159/000334318

12. Liberati A, Altman DG, Tetzlaff J, Mulrow C, Gøtzsche PC, loannidis JP, et al. The PRISMA statement for reporting systematic reviews and meta-analyses of studies that evaluate health care interventions: explanation and elaboration. J Clin Epidemiol. 2009 Oct;62(10):e1-34. https://doi.org/10.1016/j.jclinepi.2009.06.006

13. Valente LL, Sarkis-Onofre R, Gonçalves AP, Fernández E, Loomans B, Moraes RR. Repair bond strength of dental composites: systematic review and meta-analysis. Int J Adhes Adhes. 2016;69:15-26. https://doi.org/10.1016/i.ijadhadh.2016.03.020

14. Abdelwahed AG, Temirek MM, Hassan FM. Antierosive effect of topical fluorides: a systematic review and meta-analysis of in situ studies. Open Access Maced J Med Sci. 2019 May;7(9):1523-30. https://doi.org/10.3889/oamims.2019.291

15. Higgins J, Thomas J, Chandler J, Cumpston M, Li T, Page M, Welch V, editors. Cochrane handbook for systematic reviews of interventions Version 6.1. London: Cochrane; 2020.

16. Schlueter N, Hara A, Shellis RP, Ganss C. Methods for the measurement and characterization of erosion in enamel and dentine. Caries Res. 2011;45(1 Suppl 1):13-23. https://doi.org/10.1159/000326819

17. Hara AT, Lippert F, Zero DT. Interplay between experimental dental pellicles and stannous-containing toothpaste on dental erosion-abrasion. Caries Res. 2013;47(4):325-9. https://doi.org/10.1159/000347051 
Reduction in erosive tooth wear using stannous fluoride-containing dentifrices: a meta-analysis

18. Hara AT, González-Cabezas C, Creeth J, Parmar M, Eckert GJ, Zero DT. Interplay between fluoride and abrasivity of dentifrices on dental erosion-abrasion. J Dent. 2009 Oct;37(10):781-5. https://doi.org/10.1016/i.jdent.2009.06.006

19. Wiegand A, Egert S, Attin T. Toothbrushing before or after an acidic challenge to minimize tooth wear? An in situ/ex vivo study. Am J Dent. 2008 Feb;21(1):13-6. https://doi.org/10.5167/uzh-6592

20. Murad MH, Montori VM, loannidis JP, et al. Fixed-effects and random-effects models. In: Guyatt G, Rennie D, Meade MO, Cook DJ, editors. Users' guide to the medical literature: a manual for evidence-based clinical practice. 3rd ed. New York: McGraw-Hill; 2015.

21. Tufanaru C, Munn Z, Stephenson M, Aromataris E. Fixed or random effects meta-analysis? Common methodological issues in systematic reviews of effectiveness. Int J Evid-Based Healthc. 2015 Sep;13(3):196-207. https://doi.org/10.1097/XEB.0000000000000065

22. Paez A. Grey literature: an important resource in systematic reviews. J Evid Based Med. 2017 Dec;10(3):233-40. https://doi.org/10.1111/jebm.12266

23. Oliveira MAM, Torres CP, Gomes-Silva JM, Chinelatti MA, Menezes FC, Palma-Dibb RG, et al. Microstructure and mineral composition of dental enamel of permanent and deciduous teeth. Microsc Res Tech. 2010 May;73(5):572-7. https://doi.org/10.1002/jemt.20796

24. Teruel JD, Alcolea A, Hernández A, Ruiz AJ. Comparison of chemical composition of enamel and dentine in human, bovine, porcine and ovine teeth. Arch Oral Biol. 2015 May;60(5):768-75. https://doi.org/10.1016/i.archoralbio.2015.01.014

25. Fonseca RB, Haiter-Neto F, Carlo HL, Soares CJ, Sinhoreti MA, Puppin-Rontani RM, et al. Radiodensity and hardness of enamel and dentin of human and bovine teeth, varying bovine teeth age. Arch Oral Biol. 2008 Nov;53(11):1023-9. https://doi.org/10.1016/i.archoralbio.2008.06.012

26. Hara AT, Turssi CP, Teixeira EC, Serra MC, Cury JA. Abrasive wear on eroded root dentine after different periods of exposure to saliva in situ. Eur J Oral Sci. 2003 Oct;111(5):423-7. https://doi.org/10.1034/i.1600-0722.2003.00074.x 